\title{
MicroRNA expression profiles in supraglottic carcinoma
}

\author{
TIANHONG ZHANG ${ }^{1 *}$, GUOJUN HAN $^{1 *}$, YANSONG WANG $^{3}$, KEWA CHEN $^{1}$ and YANAN SUN ${ }^{2}$ \\ ${ }^{1}$ Department of Otorhinolaryngology, Head and Neck Surgery, The First Affiliated Hospital, Harbin Medical University; \\ ${ }^{2}$ Department of Otorhinolaryngology, Head and Neck Surgery, The Second Affiliated Hospital, \\ Harbin Medical University; ${ }^{3}$ Department of Anesthesiology, The First Affiliated Hospital, \\ Harbin Medical University, Harbin, Heilongjiang 150001, P.R. China
}

Received October 14, 2013; Accepted November 27, 2013

DOI: $10.3892 /$ or.2014.3117

\begin{abstract}
MicroRNAs (miRNAs) are single-stranded RNA molecules which regulate gene expression at the post-transcriptional level and several miRNAs have been found to be associated with some types of cancer. We sought to identify the expression and involvement of miRNAs in supraglottic carcinoma tissues compared with normal tissues and to determine whether miRNA expression is predictive of disease. Unsupervised clustering shows that miRNA profiles can distinguish tumor from normal tissues. Analysis of miRNA contents in supraglottic carcinoma highlighted nineteen differentially expressed miRNAs, three upregulated miRNAs (miR-21, miR-19a, miR-33a) and two downregulated miRNAs (miR-206, miR-375). The microarray results of supraglottic carcinoma and related computer analysis may be beneficial for further analysis of cancer diagnosis and therapy.
\end{abstract}

\section{Introduction}

Laryngeal squamous cell carcinoma (LSCC) is a very common malignant neoplasm of the head and neck. One of the key challenges in the treatment of LSCC is the management of metastasis to locoregional lymph nodes. Lymph node metastasis is common in supraglottic carcinoma and is the main cause of mortality for these patients. It has been reported that the incidence of lymph node metastasis may be as high as $25-50 \%$ in supraglottic carcinoma (1). Current treatments, including surgical intervention, radiation therapy and

Correspondence to: Dr Tianhong Zhang, Department of Otorhinolaryngology, Head and Neck Surgery, The First Affiliated Hospital, Harbin Medical University, Harbin, Heilongjiang 150001, P.R. China E-mail: tianhongzhangcn@126.com

Dr Yanan Sun, Department of Otorhinolaryngology, Head and Neck Surgery, The Second Affiliated Hospital, Harbin Medical University, Harbin, Heilongjiang 150001, P.R. China

E-mail: yanansuna@163.com

*Contributed equally

Key words: microRNAs, supraglottic carcinoma, microarray analysis, cancer chemotherapy, have a moderate effect on early-stage cases, but are less effective in more advanced cases. Five-year overall survival for supraglottic cancer remains poor $(2,3)$. Therefore, understanding the molecular pathways of carcinogenesis or progression is key to improving diagnosis, therapy and prevention of supraglottic carcinoma.

microRNAs (miRNAs) are a class of small, non-coding RNAs that are endogenously expressed in animal and plant cells. They regulate the expression of protein-coding genes at the translational level. One strand of the mature double-stranded miRNA is incorporated into the RNA-induced silencing complex, which downregulates target mRNAs either by degradation or by translational inhibition (4). miRNAs play important roles in normal regulation of gene expression for developmental timing, cell proliferation and apoptosis. Moreover, altered miRNA expression is implicated in cancer. Recently, miRNA genes were implicated in several types of cancer (5-7). The expression of miRNAs varies between cancer and normal cells and it also varies among different types of cancer. It has been shown that some miRNAs are aberrantly expressed in several different types of cancer (8-10), suggesting that they may play a role as a novel class of oncogenes or tumor-suppressor genes.

Several target genes have been experimentally identified for some miRNAs in various LSCCs; however, the global pattern of cellular functions and pathways affected by miRNAs in supraglottic cancer remains elusive. In the present study, expression profiling of miRNAs in clinical supraglottic carcinoma tissue samples was carried out, revealing the relationship between miRNA expression and supraglottic carcinoma. We also studied the candidate expression of genes that regulate supraglottic carcinoma miRNA processing and identified the possible gene ontology, pathway, evolution relationship of these candidate target genes. This information may be the basis of identifying a clinically applicable diagnostic tool in the future.

\section{Materials and methods}

Laryngeal carcinoma specimens and RNA isolation. Ten supraglottic carcinoma specimens and ten adjacent normal tissues were collected by surgical resection from the First Affiliated Hospital of Harbin Medical University between January and December 2008. These specimens were from patients between 42 to 78 years old, including 6 males and 
4 females. Lymph metastasis was found in three cases. Prior to surgery, no patients were treated by radiotherapy or chemotherapy and they were in good condition without cancer transmission found from other parts of their bodies.

The samples were snap-frozen in liquid nitrogen and stored at $-80^{\circ} \mathrm{C}$. Total RNA was isolated from adjacent normal tissue and tumor tissues using the TRIzol reagent (Invitrogen, Carlsbad, CA, USA) according to the manufacturer's protocol. RNA was quantified using the BioPhotometer (Eppendorf), aliquoted, and stored at $-80^{\circ} \mathrm{C}$ briefly until needed.

Microarray profiling. miRNA was synthesized, amplified and purified using the Illumina TotalPrep RNA Amplification kit (Ambion Inc.), following the manufacturer's recommendations. Total RNA (500 ng) was sent for miRNA profiling studies using the Human miRNA BeadChip (V.12.0) in Illumina BeadStation 500GX (Illumina, Inc.), which is single-channel format according to the standard operating procedures of the company. RNA was reverse transcribed. After second strand synthesis, the cDNA was transcribed in vitro and ncRNA labelled with biotin-16-UTP. Labelled probe hybridization to BeadChip was carried out. Briefly, poly-A tail was added, cDNA synthesis (biotinylated) followed, combined with miRNA specific oligo, then extension and ligation. PCR (Mastercycler 5333), lable, hybridization $\left(45^{\circ} \mathrm{C}, 14-20 \mathrm{~h}\right)$, wash and imaging were carried our. Human miRNA BeadChip (V.12.0) contains 1,145 probes specific to human miRNA assessed at $\sim 30$ different beads on average ( $\sim 850$ probes from Sanger miRBase V.12.0 and 296 from literature or novel content identified with Illumina Solexa sequencing). The $\mathrm{Cy} 3$ fluorescence on the arrays was scanned at an excitation wavelength of $532 \mathrm{~nm}$ using a BeadArray Reader GX scanner (Illumina). Illumina BeadStudio software (version 1.5.0.34) was used for preliminary data analysis. Several quality control procedures were implemented to assess the quality of the whole experiment. Total RNA control samples were analyzed in the process. The Illumina BeadStudio software was used to view control summary reports and scatter plots. The scatter plots indicated a reduction in assay performance and highlighted samples that were of lower quality. The control summary report is generated by the BeadStudio software, which evaluates the performance of the built-in controls of the BeadChips in the process. This allows the user to look for variations in signal intensity, hybridization signal, background signal and the background to noise ratio for all samples analyzed in that run. Data are expressed as $\log 2$ ratios of fluorescence intensities of the experimental and the common reference sample. The Illumina data were then normalized using the 'normalize quantiles' function in the BeadStudio software.

miRNA expression analysis. Cluster analysis was performed using the metric Euclidean distance to compute the distance matrix for supraglottic carcinoma patients based on the expression of 1,145 differentially expressed miRNAs. To agglomerate the patients in the hierarchical cluster, we used the Ward method. Function heatmap. 2 from R package gplots was used for the graphical display of the dendrogram (11).

Predicted miRNA target analysis. Potential miRNA targets were predicted and analyzed using DIANA TarBase (12) (http://
Table I. Differentially expressed miRNAs in supraglottic carcinoma tissues compared with normal tissues.

Expression fold

miRNA Chromosome (cancer/normal tissue) P-value

\begin{tabular}{lrcc}
\hline miR-34b & 11 & 0.061481 & $7.36 \mathrm{E}-38$ \\
miR-885-5p & 3 & 0.107502 & $7.36 \mathrm{E}-38$ \\
miR-218 & 4,5 & 0.133269 & $7.36 \mathrm{E}-38$ \\
miR-34c-3p & 11 & 0.133316 & $7.36 \mathrm{E}-38$ \\
miR-299-5p & 14 & 0.134148 & $7.36 \mathrm{E}-38$ \\
miR-375 & 2 & 0.139267 & $7.36 \mathrm{E}-38$ \\
miR-30a & 6 & 0.140864 & $3.62 \mathrm{E}-33$ \\
miR-206 & 6 & 0.148176 & $2.83 \mathrm{E}-10$ \\
miR-410 & 14 & 0.16099 & $2.15 \mathrm{E}-27$ \\
miR-1299 & 9 & 0.16614 & $6.60 \mathrm{E}-25$ \\
miR-128a:9.1 & - & 0.168493 & $7.36 \mathrm{E}-38$ \\
miR-432 & 14 & 0.170929 & $6.93 \mathrm{E}-37$ \\
miR-136 & 14 & 0.173921 & $6.49 \mathrm{E}-13$ \\
miR-486-3p & 8 & 0.182158 & $1.16 \mathrm{E}-36$ \\
miR-337-3p & 14 & 0.186334 & $7.86 \mathrm{E}-35$ \\
miR-125b-2 & 21 & 0.187213 & $3.62 \mathrm{E}-20$ \\
miR-21 & 17 & 8.453834 & 0.008087 \\
miR-19a & 13 & 13.15016 & 0.0054576 \\
miR-33a & 22 & 13.33146 & 0.0057371 \\
\end{tabular}

The miRNA microarray analysis was performed to detect the differentially expressed miRNAs in supraglottic carcinoma and normal tissues. P-values were generated in BeasStudio Software. miRNA, microRNA.

diana.pcbi.upenn.edu/). Predicted target lists were analyzed using DIANA mirPath for association with molecular pathways potentially altered by the expression of single or multiple miRNAs (13). Predicted target lists were also analyzed for association with Gene Ontology (GO) (14) terms using L2L microarray analysis tools (15).

Evolution analysis. Potential miRNA target sequences were obtained from the National Center for Biotechnology Information website (NCBI, USA). The sequences were aligned by the ClustalW program (http://www.ebi.ac.uk/ clustalw/) (16). The evolutionary tree of protein sequences was constructed by the neighbor-joining (NJ) method with protein p-distances by MEGA4 (Molecular Evolutionary Genetics Analysis) software (http://www.megasoftware.net/index. html) (17) and the reliability of the tree topology was assessed by 1,000 bootstrap replications.

\section{Results}

miRNA expression profile in supraglottic carcinoma and normal tissues. Using the miRNA microarray containing 1,145 human miRNA probe sets, we first assessed the miRNA profiles in three paired supraglottic carcinoma and normal tissues.

Unsupervised hierarchical clustering based on all the miRNAs spotted on the chip revealed a marked, distinct 


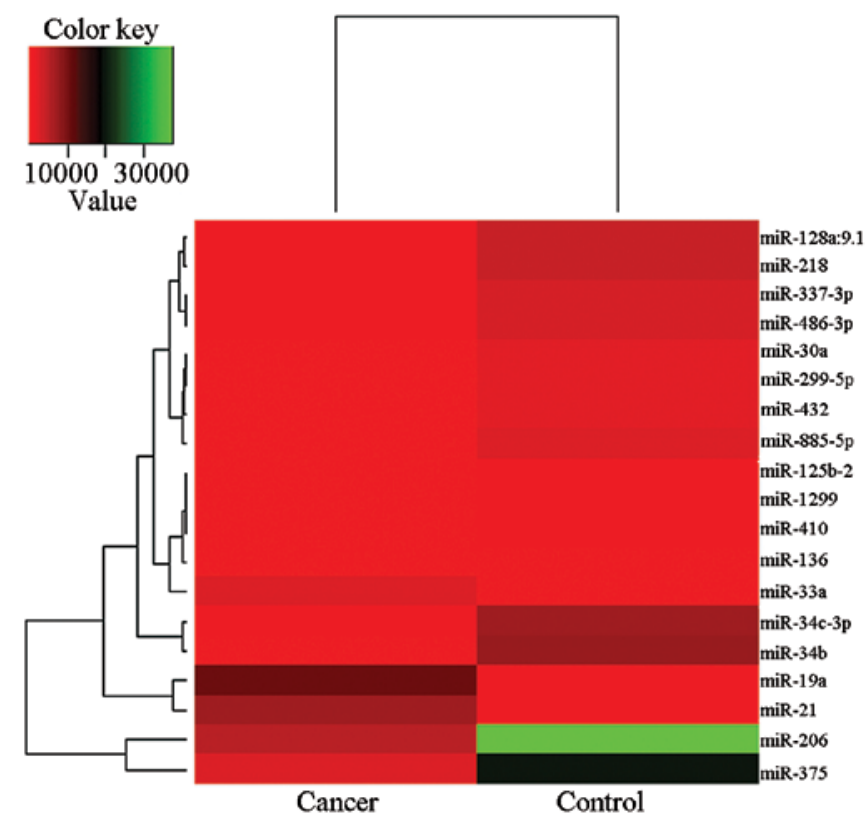

Figure 1. Differentially expressed miRNAs between supraglottic carcinoma and normal tissues. Results of the unsupervised hierarchical clustering analysis between groups of cancer tissues and normal tissues based on differential expression of 19 significantly altered miRNAs. The heat map shows relative levels of miRNA expression in a red (low relative expression) to green (high relative expression) scale. miRNA, microRNA.

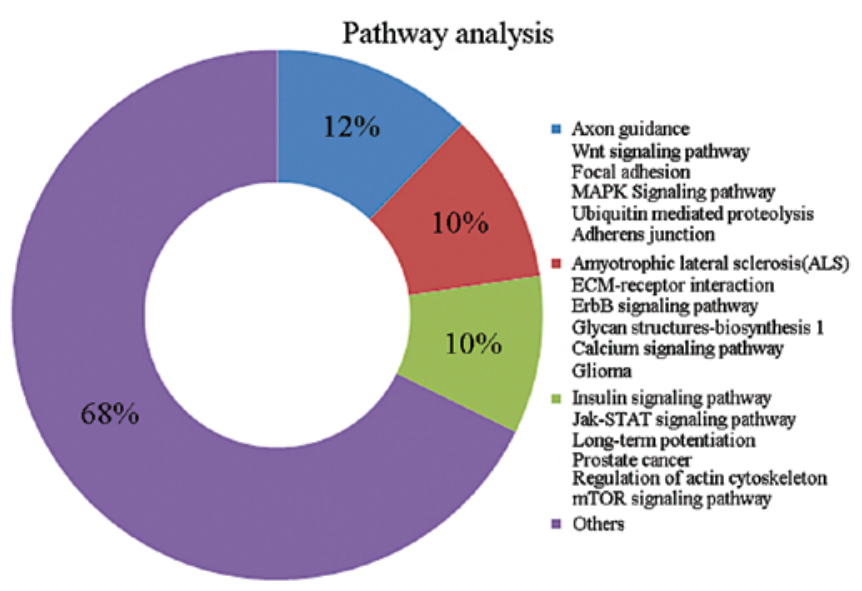

Figure 2. Potential miRNA target gene pathway analysis in supraglottic carcinoma. miRNA, microRNA.

separation of the supraglottic carcinoma miRNA profiles compared with those of normal tissues. Significance analysis of microarray identified 85 significantly differentially expressed miRNAs $(\mathrm{P}<0.01)$ between supraglottic carcinoma and normal tissues. These human miRNAs further identified by significance analysis of microarray method showed $>5$-fold difference between supraglottic carcinoma and normal tissues. This method identified 19 miRNAs to be significantly altered in their expression between supraglottic carcinoma and non-diseased tissues, with 3 being upregulated (miR-21, miR-19a, miR-33a) and 16 downregulated in supraglottic carcinoma (Table I). Unsupervised clustering analysis using these 19 miRNAs discriminated cancer from non-cancer tissues (Fig. 1), thus indicating that these miRNAs may be a

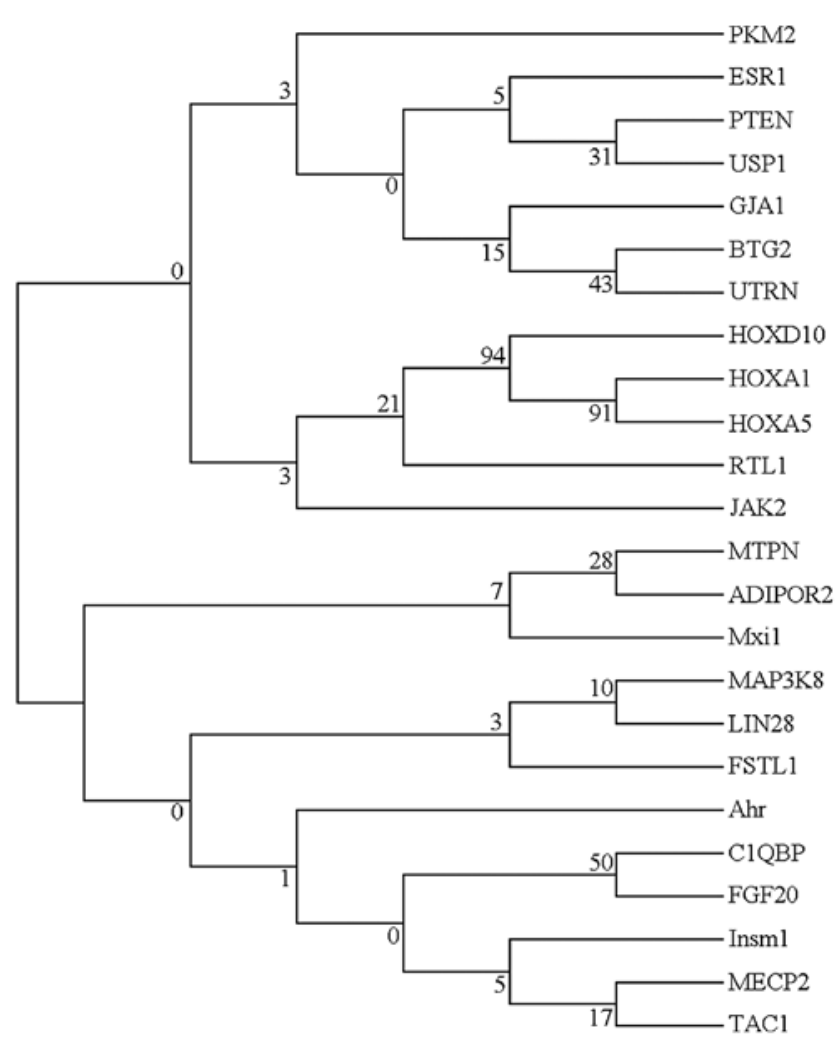

Figure 3. Evolutionary tree and sequence divergences of potential miRNA target genes. The values on each node are percentage of bootstrap values. miRNA, microRNA.

valid diagnostic for supraglottic carcinoma, although due to the small number of samples analyzed, this requires further analysis.

Bioinformatics analysis of supraglottic carcinoma miRNA target genes. To investigate the involvement of miRNAs in supraglottic carcinoma, we performed systemic bioinformatics analysis to identify potential gene targets, including pathway analysis (Fig. 2). Several pathways were enriched in the target genes. Notably, multiple genes were related to signaling pathways. The list of regulated gene targets was also used to perform a GO (14) analysis to evaluate the specific functional categories of genes from broad GO categories (Table II). The results indicated that seven biological processes, six molecular functions and two cellular components were statistically enriched with these miRNA targets. Furthermore, we found that 25 potential target genes related to 12 miRNAs which were strongly supported by references to avoid the false negative predictions (Table III). As a result, 3 target genes were involved in mRNA cleavage and 13 target genes were involved in mRNA repression. The evolutionary tree of miRNA target genes was further analyzed to illustrate the function of these genes (Fig. 3). These genes showed a high level of sequence divergence. It is reasonable to infer that these genes are members of a multigene family, since they shared low sequence similarity. Generally, a node with $>70 \%$ bootstrap supporting value is a stable node. The NJ-tree of miRNA target genes exhibited low bootstrap supporting values on each deep branch node and, therefore, few strong conclusions may be drawn from the evolutionary tree. 
Table II. Gene Ontology analysis in supraglottic carcinoma.

\begin{tabular}{|c|c|c|c|}
\hline GO type & GO term & Description & P-value \\
\hline Process & GO: 0006725 & Cellular aromatic compound metabolic process & 2.79E-4 \\
\hline Process & GO: 0050776 & Regulation of immune response & 4.64E-4 \\
\hline Process & GO: 0001919 & Regulation of receptor recycling & 4.94E-4 \\
\hline Process & GO: 0003015 & Heart process & $5 \mathrm{E}-4$ \\
\hline Process & GO: 0060047 & Heart contraction & $5 \mathrm{E}-4$ \\
\hline Process & GO: 0019882 & Antigen processing and presentation & $5.76 \mathrm{E}-4$ \\
\hline Process & GO: 0006066 & Alcohol metabolic process & $8.35 \mathrm{E}-4$ \\
\hline Process & GO: 0008152 & Metabolic process & $9.43 \mathrm{E}-4$ \\
\hline Process & GO: 0031943 & Regulation of glucocorticoid metabolic process & $9.83 \mathrm{E}-4$ \\
\hline Function & GO: 0005488 & Binding & $5.05 \mathrm{E}-7$ \\
\hline Function & GO: 0003824 & Catalytic activity & $1.08 \mathrm{E}-5$ \\
\hline Function & GO: 0004423 & Iduronate-2-sulfatase activity & $5.37 \mathrm{E}-4$ \\
\hline Function & GO: 0046872 & Metal ion binding & $5.9 \mathrm{E}-4$ \\
\hline Function & GO: 0000062 & Acyl-CoA binding & $9.21 \mathrm{E}-4$ \\
\hline Function & GO: 0043169 & Cation binding & $9.35 \mathrm{E}-4$ \\
\hline Component & GO: 0044424 & Intracellular part & $3.63 \mathrm{E}-6$ \\
\hline Component & GO: 0044444 & Cytoplasmic part & $8.87 \mathrm{E}-5$ \\
\hline
\end{tabular}

Gene Ontology (GO) analysis was performed to detect the differentially expressed miRNAs in supraglottic carcinoma and normal tissues. P-values were calculated from binomial distribution. miRNAs, microRNAs.

Table III. Comparison of pathways associated with miRNA and mRNA signatures.

\begin{tabular}{|c|c|c|c|}
\hline miRNA & Gene & Type & Author/(Ref.) \\
\hline miR-10a & HOXA1 & mRNA repression & Garzon et al (29) \\
\hline miR-10b & HOXD10 & mRNA repression & Ma et al (33) \\
\hline $\operatorname{miR}-125 b$ & LIN28 & mRNA cleavage & Wu and Belasco (39) \\
\hline miR-133a & PKM2 & Unknown & Wong et al (38) \\
\hline $\operatorname{miR}-133 b$ & PKM2 & Unknown & Wong et al (38) \\
\hline $\operatorname{miR}-136$ & RTL1 & mRNA cleavage & Davis et al (28) \\
\hline miR-19a & MECP2 & Unknown & Lewis et al (32) \\
\hline miR-19a & PTEN & mRNA repression & Lewis et al (32) \\
\hline miR-19a & HOXA5 & Unknown & Lewis et al (32) \\
\hline miR-206 & FSTL1 & mRNA repression & Rosenberg et al (36) \\
\hline miR-206 & UTRN & mRNA repression & Rosenberg et al (36) \\
\hline miR-206 & GJA1 & mRNA repression & Anderson et al (27) \\
\hline miR-206 & ESR1 & mRNA cleavage & Adams et al (26) \\
\hline miR-206 & TAC1 & mRNA repression & Greco and Rameshwar (30) \\
\hline miR-370 & MAP3K8 & Unknown & Meng et al (34) \\
\hline miR-375 & MTPN & mRNA repression & Poy et al (35) \\
\hline miR-375 & Ahr & Unknown & Krek et al (31) \\
\hline miR-375 & C1QBP & mRNA repression & Krek et al (31) \\
\hline miR-375 & Insm1 & Unknown & Krek et al (31) \\
\hline miR-375 & ADIPOR2 & mRNA repression & Krek et al (31) \\
\hline miR-375 & JAK2 & mRNA repression & Krek et al (31) \\
\hline miR-375 & Mxi1 & Unknown & Krek et al (31) \\
\hline miR-375 & USP1 & mRNA repression & Krek et al (31) \\
\hline miR-433 & FGF20 & Unknown & Wang et al (37) \\
\hline miR-21 & BTG2 & mRNA repression & Liu et al (22) \\
\hline
\end{tabular}

Pathway analysis was performed to detect the differentially expressed miRNAs in supraglottic carcinoma and normal tissues. miRNAs, microRNAs. 


\section{Discussion}

The present study demonstrated that multiple microRNAs (miRNAs) are upregulated or downregulated in supraglottic carcinoma, including an extensively validated subset that may be potential clinical biomarkers of disease. Microarray profiling of more than 1,000 miRNAs identified 85 miRNAs that were significantly differentially expressed in tumor tissues compared with normal tissues. Of the 85 human miRNAs, three upregulated miRNAs (miR-21, miR-19a, miR-33a) and two downregulated miRNAs (miR-206, miR-375) were highlighted. Twenty-five target genes were identified to help characterize the diverse functions of miRNAs. Furthermore, biology process and evolution were explored to find the function of miRNAs.

miRNA expression profiles can be used to classify human cancer $(14,18)$. Distinct signatures for several epithelial cancers have been reported, such as breast, lung, pancreatic and gastric cancer $(9,19-21)$. Our study explored miRNA expression in supraglottic carcinoma. Consistent with previous reports of miRNA expression in head and neck cancer (22-25), some miRNAs were significantly deregulated, although changes in individual miRNAs did not match completely. For example, miR-21 was significantly upregulated in all head and neck cancer analysis (22-25). Tran et al found miR-21 and miR-205 were highly expressed in head and neck cancer cell lines (25). Chang et al found miR-21, let-7, 18, 29c, 142-3p, 155 and 16b were significantly overexpressed in primary head and neck squamous cell carcinoma (24). Avissar et al found miR-221 and miR-375 were significantly altered and upregulated in head and neck squamous cell carcinoma (23). Liu et al identified 13 miRNAs that were differentially expressed, 7 miRNAs were downregulated and 6 miRNAs (let-7a-1, miR-203, miR-205, miR-21, miR-98 and miR-16-1) were upregulated in laryngeal carcinoma tissues based on 210 human miRNA probe sets (22). This indicated that the molecular biology of head and neck squamous cell carcinoma is complex. It is important to analyze the miRNA mechanism on a larger scale.

miRNAs modulate gene expression by targeting mRNAs for translational suppression or mRNA cleavage and it is well known that miRNAs regulate a variety of cellular activities through their effect on the expression of multiple target genes (4). The identification of target genes regulated by a specific miRNA has been proved difficult despite the development of computational approaches to predict miRNA targets. The ability to find target genes is further complicated by the fact that target selectivity of miRNAs may depend on the cellular microenvironment. Our studies identified 25 reliable target genes, supported by previous studies $(22,26-39)$. The evolutionary tree of these target genes showed a low sequence similarity and it indicated that miRNAs may play different roles in supraglottic carcinoma. Also, these results provided a possible way to address biological meaning from the global pattern of cellular functions and pathways that are affected by miRNAs in supraglottic carcinoma. Gene Ontology (GO) (14) was developed into three structured controlled vocabularies (ontologies) that describe gene products in components and molecular functions in a species-independent manner (40). $\mathrm{GO}$ enrichment analysis was used to reduce the number of targets of a large group of co-expressed miRNAs and to find biological functions potentially affected by multiple miRNAs in our research. We performed a statistical enrichment analysis of $\mathrm{GO}$ categories to find categories that are enriched with targets of co-expressed miRNAs. It allowed us to reduce a very large raw list of predicted target genes to a smaller set of target genes from significantly enriched GO categories. We assumed that filtering GO categories on the total number of hits by miRNAs targeting the same category would reduce the number of false positive target predictions and at the same time would allow narrowing down of the large target lists and determining those biological functions.

In conclusion, our global analysis of miRNA array in human supraglottic carcinoma tissues demonstrated that co-expressed miRNAs may collectively provide systemic compensatory response to the abnormal functional and phenotypic changes in supraglottic carcinoma by targeting a broad range of functional categories and abnormally activated pathways known to be affected in supraglottic carcinoma. Such system biology based approach may provide new avenues for biological interpretation of miRNA profiling data as well as generation of experimentally testable hypotheses regarding collective regulatory functions of miRNAs in supraglottic carcinoma biology.

\section{Acknowledgements}

This study was supported by grants from the Heilongjiang Postdoctoral Foundation (LBHZ12194), and the Research Foundation of Heilongjiang Provincial Department of Education.

\section{References}

1. Rudolph E, Dyckhoff G, Becher H, Dietz A and Ramroth H: Effects of tumour stage, comorbidity and therapy on survival of laryngeal cancer patients: a systematic review and a metaanalysis. Eur Arch Otorhinolaryngol 268: 165-179, 2011.

2. Cosetti M, Yu GP and Schantz SP: Five-year survival rates and time trends of laryngeal cancer in the US population. Arch Otolaryngol Head Neck Surg 134: 370-379, 2008.

3. Petrakos I, Kontzoglou K, Nikolopoulos TP, Papadopoulos O and Kostakis A: Glottic and supraglottic laryngeal cancer: epidemiology, treatment patterns and survival in 164 patients. J BUON 17: 700-705, 2012.

4. Bartel DP: MicroRNAs: genomics, biogenesis, mechanism, and function. Cell 116: 281-297, 2004.

5. Ferretti E, De Smaele E, Po A, et al: MicroRNA profiling in human medulloblastoma. Int J Cancer 124: 568-577, 2009.

6. Khoshnaw SM, Green AR, Powe DG and Ellis IO: MicroRNA involvement in the pathogenesis and management of breast cancer. J Clin Pathol 62: 422-428, 2009.

7. Raponi M, Dossey L, Jatkoe T, et al: MicroRNA classifiers for predicting prognosis of squamous cell lung cancer. Cancer Res 69: 5776-5783, 2009.

8. Akao Y, Nakagawa Y and Naoe T: MicroRNAs 143 and 145 are possible common onco-microRNAs in human cancers. Oncol Rep 16: 845-850, 2006.

9. Iorio MV, Ferracin M, Liu CG, et al: MicroRNA gene expression deregulation in human breast cancer. Cancer Res 65: 7065-7070, 2005.

10. Michael MZ, O' Connor SM, van Holst Pellekaan NG, Young GP and James RJ: Reduced accumulation of specific microRNAs in colorectal neoplasia. Mol Cancer Res 1: 882-891, 2003.

11. Gentleman RC, Carey VJ, Bates DM, et al: Bioconductor: open software development for computational biology and bioinformatics. Genome Biol 5: R80, 2004.

12. Papadopoulos GL, Reczko M, Simossis VA, Sethupathy P and Hatzigeorgiou AG: The database of experimentally supported targets: a functional update of TarBase. Nucleic Acids Res 37: D155-D158, 2009. 
13. Papadopoulos GL, Alexiou P, Maragkakis M, Reczko M and Hatzigeorgiou AG: DIANA-mirPath: integrating human and mouse microRNAs in pathways. Bioinformatics 25: 1991-1993, 2009.

14. Lu J, Getz G, Miska EA, et al: MicroRNA expression profiles classify human cancers. Nature 435: 834-838, 2005.

15. Newman JC and Weiner AM: L2L: a simple tool for discovering the hidden significance in microarray expression data. Genome Biol 6: R81, 2005.

16. Thompson JD, Higgins DG and Gibson TJ: CLUSTAL W: improving the sensitivity of progressive multiple sequence alignment through sequence weighting, position-specific gap penalties and weight matrix choice. Nucleic Acids Res 22: 4673-4680, 1994.

17. Kumar S, Nei M, Dudley J and Tamura K: MEGA: a biologistcentric software for evolutionary analysis of DNA and protein sequences. Brief Bioinform 9: 299-306, 2008.

18. Volinia S, Calin GA, Liu CG, et al: A microRNA expression signature of human solid tumors defines cancer gene targets. Proc Natl Acad Sci USA 103: 2257-2261, 2006.

19. Petrocca F, Visone R, Onelli MR, et al: E2F1-regulated microRNAs impair TGF $\beta$-dependent cell-cycle arrest and apoptosis in gastric cancer. Cancer Cell 13: 272-286, 2008.

20. Bloomston M, Frankel WL, Petrocca F, et al: MicroRNA expression patterns to differentiate pancreatic adenocarcinoma from normal pancreas and chronic pancreatitis. JAMA 297: 1901-1908, 2007.

21. Yanaihara N, Caplen N, Bowman E, et al: Unique microRNA molecular profiles in lung cancer diagnosis and prognosis. Cancer Cell 9: 189-198, 2006.

22. Liu M, Wu H, Liu T, et al: Regulation of the cell cycle gene, $B T G 2$, by miR-21 in human laryngeal carcinoma. Cell Res 19 828-837, 2009.

23. Avissar M,Christensen BC, Kelsey KT and Marsit CJ: MicroRNA expression ratio is predictive of head and neck squamous cell carcinoma. Clin Cancer Res 15: 2850-2855, 2009.

24. Chang SS, Jiang WW, Smith I, et al: MicroRNA alterations in head and neck squamous cell carcinoma. Int J Cancer 123 2791-2797, 2008

25. Tran N, McLean T, Zhang X, et al: MicroRNA expression profiles in head and neck cancer cell lines. Biochem Biophys Res Commun 358: 12-17, 2007.

26. Adams BD, Furneaux $\mathrm{H}$ and White BA: The micro-ribonucleic acid (miRNA) miR-206 targets the human estrogen receptor- $\alpha$ $(\mathrm{ER} \alpha)$ and represses ER $\alpha$ messenger RNA and protein expression in breast cancer cell lines. Mol Endocrinol 21: 1132-1147, 2007.
27. Anderson C, Catoe $\mathrm{H}$ and Werner R: MIR-206 regulates connexin43 expression during skeletal muscle development. Nucleic Acids Res 34: 5863-5871, 2006.

28. Davis E, Caiment F, Tordoir X, et al: RNAi-mediated allelic trans-interaction at the imprinted Rtll/Peg11 locus. Curr Biol 15: 743-749, 2005.

29. Garzon R, Pichiorri F, Palumbo T, et al: MicroRNA fingerprints during human megakaryocytopoiesis. Proc Natl Acad Sci USA 103: 5078-5083, 2006.

30. Greco SJ and Rameshwar P: MicroRNAs regulate synthesis of the neurotransmitter substance $\mathrm{P}$ in human mesenchymal stem cell-derived neuronal cells. Proc Natl Acad Sci USA 104: 15484-15489, 2007.

31. Krek A, Grün D, Poy MN, et al: Combinatorial microRNA target predictions. Nat Genet 37: 495-500, 2005.

32. Lewis BP, Shih IH, Jones-Rhoades MW, Bartel DP and Burge CB: Prediction of mammalian microRNA targets. Cell 115: 787-798, 2003.

33. Ma L, Teruya-Feldstein $\mathbf{J}$ and Weinberg RA: Tumour invasion and metastasis initiated by microRNA-10b in breast cancer. Nature 449: 682-688, 2007.

34. Meng F, Wehbe-Janek H, Henson R, Smith H and Patel T: Epigenetic regulation of microRNA-370 by interleukin-6 in malignant human cholangiocytes. Oncogene 27: 378-386, 2008.

35. Poy MN, Eliasson L, Krutzfeldt J, et al: A pancreatic islet-specific microRNA regulates insulin secretion. Nature 432: 226-230, 2004.

36. Rosenberg MI, Georges SA, Asawachaicharn A, Analau E and Tapscott SJ: MyoD inhibits Fstl1 and Utrn expression by inducing transcription of miR-206. J Cell Biol 175: 77-85, 2006.

37. Wang G, van der Walt JM, Mayhew G, et al: Variation in the miRNA-433 binding site of FGF20 confers risk for Parkinson disease by overexpression of $\alpha$-synuclein. Am J Hum Genet 82: 283-289, 2008

38. Wong TS, Liu XB, Chung-Wai Ho A, et al: Identification of pyruvate kinase type $\mathrm{M} 2$ as potential oncoprotein in squamous cell carcinoma of tongue through microRNA profiling. Int J Cancer 123: 251-257, 2008

39. Wu L and Belasco JG: Micro-RNA regulation of the mammalian lin-28 gene during neuronal differentiation of embryonal carcinoma cells. Mol Cell Biol 25: 9198-9208, 2005.

40. Ashburner M, Ball CA, Blake JA, et al: Gene ontology: tool for the unification of biology. The Gene Ontology Consortium. Nat Genet 25: 25-29, 2000. 